\title{
Convergencia de la trayectoria lingüística en el espacio de estados de un Controlador Difuso aplicado a un Sistema No Lineal
}

\section{Convergence of the linguistic trajectory in the state space of a Fuzzy Controller applied to a Non-linear System}

\author{
TÉLLEZ-CUEVAS, Pedro†*, HERNÁNDEZ-LUNA, Aldo y LUNA-CARRASCO, Claudia Yadira \\ Instituto Tecnológico Superior de Huauchinango
}

ID 1er Autor: Pedro, Téllez-Cuevas / ORC ID: 0000-0002-3235-1898, Researcher ID Thomson: G-2875-2019, CVU CONACYT ID: 342839

ID $1^{\text {er }}$ Coautor: Aldo, Hernández-Luna / ORC ID: 0000-0002-7717-5314, Researcher ID Thomson: I-1165-2018, CVU CONACYT ID: 441305

ID $2^{\text {do }}$ Coautor: Claudia Yadira, Luna-Carrasco / ORC ID: 0000-0002-4092-9987, Researcher ID Thomson: G-2892-2019, CVU CONACYT ID: 368419

DOI: 10.35429/JSL.2019.19.6.23.31

Recibido 23 Marzo, 2019; Aceptado 30 Junio, 2019

\begin{abstract}
Resumen
Este artículo presenta el diseño de un controlador difuso para el análisis de estabilidad basado en la convergencia de la trayectoria lingüística en el espacio de estados, para un sistema carro-péndulo invertido, el controlador difuso es del tipo Mamdani y consta de 25 reglas, 3 variables de entrada y cada una compuesta de cinco funciones de membresía. El sistema carro-péndulo invertido es representado por un modelo no lineal, del que se obtiene un modelo equivalente linealizado bajo la consideración de pequeñas oscilaciones. Los resultados se validan contra un control PID basado en la trayectoria sobre el plano de fase para evaluar la eficiencia y efectividad del controlador difuso. El comportamiento dinámico del sistema de ambos controladores se obtiene con una entrada impulso unitario, la simulación del sistema de control se desarrolla sobre el software MATLAB/SIMULINK empleando la herramienta FUZZY LOGIC TOOLBOX, la cual permite realizar pruebas y simulaciones, además de ver resultados de forma gráfica.
\end{abstract}

Control, Difuso, Estabilidad

\begin{abstract}
This article presents the design of a fuzzy controller to stability analysis base on the convergence of the linguistic trajectory in the state of space, for an inverted car-pendulum system, the fuzzy controller is of the Mamdani type and, it consists of 25 rules, 3 input variables and each one is composed of five memberships functions. The inverted car-pendulum system is represented by a non-linear model, which is obtained from a linearized equivalent model under the consideration of small oscillations. Results are validated against a PID control base on the trajectory on the phase plane to evaluate the efficiency and effectiveness of the fuzzy controller. The dynamic behavior of the system of both controllers is obtained with a unit impulse input, the simulation of the control system is developed on the MATLAB / SIMULINK software using the FUZZY LOGIC TOOLBOX, which allows to perform test and simulations, and also it shows results of graphic form.
\end{abstract}

Control, Fuzzy, Stability

Citación: TÉLlEZ-CUEVAS, Pedro, HERNÁNDEZ-LUNA, Aldo y LUNA-CARRASCO, Claudia Yadira. Convergencia de la trayectoria lingüística en el espacio de estados de un Controlador Difuso aplicado a un Sistema No Lineal. Revista de Simulación y Laboratorio. 2019, 6-19: 22-31

\footnotetext{
*Correspondencia al Autor (Correo electrónico: ingelecptc@ gmail.com)
}

$\dagger$ Investigador contribuyendo como primer Autor 


\section{Introducción}

Los sistemas de control juegan un papel fundamental en los procesos tecnológicos modernos debido a los beneficios que se obtienen a través de su uso. El desafío actual es el modelado y control, interrelacionados, de sistemas modernos y complejos, tales como los control de tráfico, procesos químicos y sistemas robóticos [1]. Entre algunas de las dificultades que presentan estas aplicaciones se encuentran las dinámicas no modeladas, las no linealidades asociadas tanto al sistema como a las incertidumbres, las perturbaciones y los cambios en los parámetros. Para controlar los sistemas antes mencionados es necesario conocer y comprender adecuadamente su funcionamiento. Una de las vías que permite llegar a dicha comprensión es el modelado matemático. Sin embargo, para simplificar el modelado, tradicionalmente se usan las versiones linealizadas de los modelos originales.

Los sistemas de control no lineal presentan resultados más exactos sobre los lineales, particularmente porque son válidos en todo rango de variación de las variables y permiten tratar incertidumbres paramétricas [2]. A diferencia de los sistemas lineales, los no lineales presentan otros fenómenos tales como: múltiples puntos de equilibrio aislados, regiones de equilibrio, ciclo límite, bifurcaciones, entre otras. Sin embargo presentan mayor complejidad en su desarrollo y análisis [3]. Un caso interesante que incluye este tipo de características es el control del péndulo invertido como sistema no lineal.

A partir de un modelo matemático se pueden aplicar técnicas convencionales de control clásico que son ampliamente utilizadas en el control de procesos, tal es el caso del control proporcional-integral-derivativo (PID), sin embargo este tipo de controlador encuentra rápidamente sus limitaciones ya que no son capaces de adaptarse a los cambios e incertidumbres sufridas por los procesos, debido a su naturaleza lineal, lo que implica que solo puede ser aplicado a sistemas lineales, en el caso del péndulo invertido, que es un sistema no lineal, no se puede aplicar directamente el control PID, a menos que el sistema sea linealizado, una opción es considerar el caso de pequeñas oscilaciones pero se limita solo al punto de equilibrio, es decir solo será efectivo en un rango aproximadamente de 0 hasta $\pm 15^{\circ}$.
En contraste con las técnicas de control clásico existen otro tipo de metodologías como la Lógica Difusa y Redes Neuronales, entre otras. Estos enfoques se utilizan como alternativas a los controladores clásicos y modernos con el fin de lograr un mejor desempeño. El Control Difuso pertenece a la familia de los sistemas no lineales, éste es empleado con éxito para controlar sistemas no lineales $y$ en donde existe una buena comprensión basada en el conocimiento del experto humano. Asimismo, al tratarse de sistemas no lineales, su comportamiento dinámico será más detallado y por consiguiente más complejo.

Dadas estas características, este trabajo está enfocado en desarrollar un controlador difuso para un sistema péndulo invertido, con el objetivo principal de evaluar las propiedades de estabilidad que presenta éste controlador, aplicado al modelo no lineal y a su versión linealizada; para éste último caso se hace una validación de los resultados obtenidos al comparar su respuesta contra la del controlador PID clásico. Es importante mencionar que este trabajo solo se enfoca en el análisis de estabilidad del controlador y se tiene planeado continuar con el análisis de robustez aplicando la norma de Hankel en el dominio de la frecuencia conocida como técnica de atenuación Hœ.

Los ejemplos más conocidos de las aplicaciones de la Inteligencia Artificial son aquellos basados en lógica difusa y redes neuronales artificiales. En los últimos años, se han desarrollado una gran cantidad de métodos de control y modelado basados en lógica difusa y redes neuronales artificiales y se han utilizado para controlar el péndulo invertido y otros sistemas complejos [4-7].

Se han aplicado estrategias de control para mover un vehículo con el péndulo invertido a bordo, utilizando transformaciones de coordenadas no lineales basadas en diseños de navegación [8]. Del mismo modo, se aplicó un control en cascada no lineal a un vehículo aéreo no tripulado para mantener el péndulo y la nave espacial estabilizada durante la trayectoria del péndulo, con el resultado de que el movimiento longitudinal del péndulo invertido es más fácil de controlar que su movimiento transversal [9]. 
En la lógica difusa una aseveración puede ser más o menos cierta, mientras que en la clásica, una aseveración es cierta o es falsa (no existen valores intermedios). La lógica difusa es una extensión de la clásica, de tal manera que permite valores intermedios entre cero y uno [10]. La lógica difusa define los conceptos en grados o variables de pertenencia, siguiendo patrones de razonamiento similares a los del pensamiento humano, una forma más práctica de enfocar los problemas como se ven en el mundo real.

Los controladores difusos se describen por reglas IF-THEN y brindan una representación sencilla y comprensible del conocimiento. El control difuso puede emplearse en innumerables aplicaciones que van desde los sistemas más sencillos, como los sistemas lineales, hasta los más complejos como los no lineales, así empleando técnicas de razonamiento aproximado es posible controlar sistemas superiores cuando el entorno no se conoce de forma precisa.

Los controladores difusos generalmente se pueden clasificar en dos tipos: Mamdani y Takagi-Sugeno [11]. La principal diferencia entre estos controladores radica en la consecuencia de las reglas. Para el controlador tipo Mamdani, esta consecuencia es un conjunto difuso y para el tipo Takagi-Sugeno es una función lineal de las entradas. En este trabajo se utiliza el tipo Mamdani. En esta clase de modelos difusos, las reglas difusas IF-THEN son de la forma siguiente:

$$
\begin{aligned}
& R^{(L)}: I F u_{1} \text { is } F_{1}^{L} A N D u_{2} \text { is } F_{2}^{L} A N D \\
& \text {.. AND } u_{P} \text { is } F_{p}^{L} \text { THEN } v \text { is } G^{L}
\end{aligned}
$$

El controlador difuso se compone principalmente de cuatro elementos, los cuales se agrupan en el sistema que se muestra en la Figura 1, y que a continuación se describen:

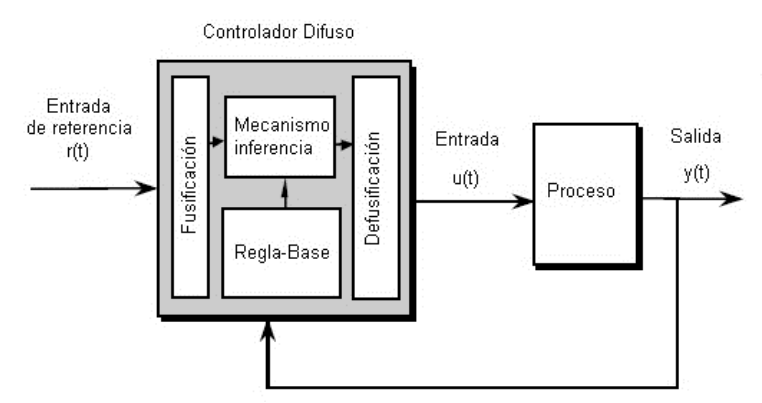

Figura 1 Diagrama de bloques de un sistema con control difuso
1. Una base-regla (es un conjunto de reglas IF - THEN), que contiene una cuantificación de la lógica difusa, es decir la descripción lingüística del experto para lograr un control deseado.

2. Un mecanismo de inferencia que emula la decisión del experto en la interpretación y aplicación de conocimientos sobre la mejor manera de controlar la planta. El mecanismo de inferencia difusa es el núcleo de cualquier controlador difuso. $\mathrm{Su}$ comportamiento dinámico es en general caracterizado por un conjunto de reglas difusas así el mecanismo de inferencia difusa es capaz de derivar una acción de control para un conjunto de valores de entrada.

3. Una interfaz de Fusificación, que convierte las variables de entrada del controlador en la información que el mecanismo de inferencia pueda usar para activar y aplicar las reglas.

4. Una interfaz de Defusificación, que convierte las conclusiones del mecanismo de inferencia en la salida real para el proceso.

El objetivo del presente trabajo es diseñar un regulador del tipo Mamdani para controlar la posición de un sistema péndulo invertido montado sobre un carro que se mueve de manera horizontal de tal forma que la varilla del péndulo se mantenga paralela al eje vertical. La metodología parte de la obtencion del modelo matematico, posteriormente el diseño de los controladores, el diseño debe incluir la validación del regulador propuesto, contra un clásico PID, además de un análisis de estabilidad basado en la convergencia de la trayectoria lingüística en el espacio de estados así como con la trayectoria en el plano de fase.

La investigación propone la tecnica, convergencia de la trayectoria lingǘstica, para para analizar el desempeño de un controlador difuso y compararlo con un metodo clasico para su validación.

\section{Metodología.}

El desarrollo de este trabajo de investigación abarca los siguientes pasos:
a. Obtencion del modelo matematico.
b. Diseño de controladores para el sistema. 
c. Simulación y analisis de respuestas de controladores del sistema péndulo invertido, ver Anexos.

d. Análisis respuestas de y estabilidad basado en la convergencia de la trayectoria lingüística, ver Resultados.

\section{Modelo Matemático Del Sistema Péndulo Invertido}

El sistema carro-péndulo invertido, como el que se muestra en la Figura 2, es de orden superior, no lineal, multivariable, rápido e inestable. Se ha convertido en un excelente sistema para llevar a cabo pruebas para diversas técnicas de control y las teorías de control moderno. Por otra parte, también puede simular muchos fenómenos en la naturaleza, tales como robots caminantes, objetos voladores en el espacio, los misiles de orientación, etc. [12].

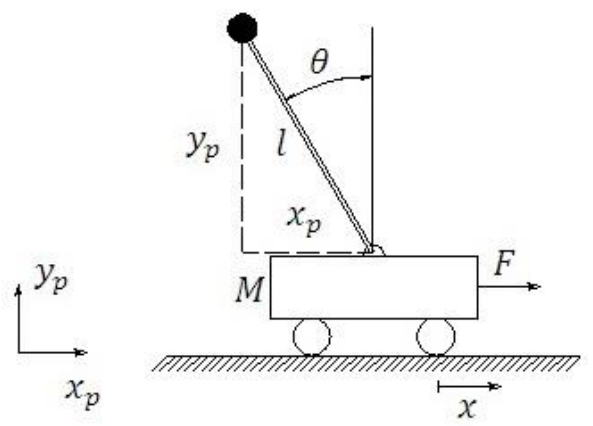

Figura 2 Sistema péndulo invertido

Para obtener el modelo matemático se requiere hacer un análisis de fuerzas, para ello se obtiene el diagrama de cuerpo libre como se muestra en la Figura 3 [12] y así determinar las fuerzas que intervengan en el sistema.
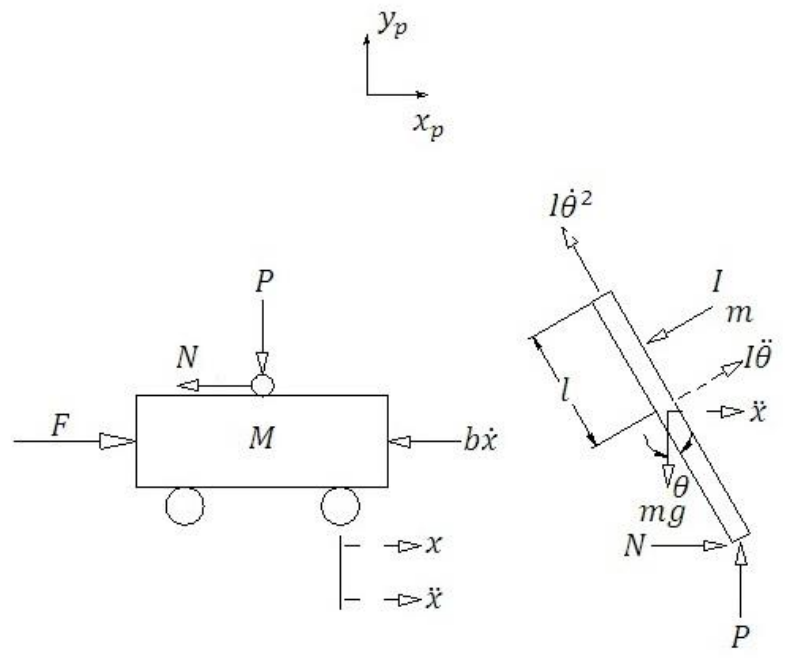

Figura 3 Diagrama de cuerpo libre
Con la ecuación de Newton que representa la suma de fuerzas para el carro y el péndulo se tienen:

$$
\begin{aligned}
& M \ddot{x}=\sum_{\text {carro }} F \\
& M \ddot{x}+b \dot{x}+N=F \\
& I \ddot{\theta}=\sum_{\text {pendulo }} T \\
& I \ddot{\theta}=N l \cos \theta+P l \operatorname{sen} \theta
\end{aligned}
$$

Donde N y P son las fuerzas de interacción entre el carro y el péndulo. Combinando las ecuaciones de fuerzas sobre el carro y el péndulo se obtienen las ecuaciones que representan el modelo matemático del sistema péndulo invertido:

$$
\begin{gathered}
(M+m) \ddot{x}+b \dot{x}+m l \dot{\theta}^{2} \operatorname{sen} \theta \\
-m l \ddot{\theta} \cos \theta=u \\
\left(I+m l^{2}\right) \ddot{\theta}+m g l \operatorname{sen} \theta=-m l \ddot{x} \cos \theta
\end{gathered}
$$

El análisis de un sistema no lineal se simplifica cuando se hace sobre su equivalente linealizado, alrededor de algún punto de operación. Sin embargo esto no es suficiente debido a que la linealización sólo predice el comportamiento local, por lo que está limitado para estudiar el comportamiento lejos del punto de operación seleccionado.

Para obtener una versión lineal del modelo del péndulo invertido se considera el caso de pequeñas oscilaciones $\left(0.261799 \mathrm{rad}=15^{\circ}\right)$, bajo estas consideraciones se tiene que: $\operatorname{sen} \theta \approx \theta \mathrm{y}$ $\cos \theta \approx 1$, por lo que las dos ecuaciones de movimiento se simplifican considerablemente como se muestran en la (8) y la (9), las cuales representan el modelo linealizado.

$(M+m) \ddot{x}+b \dot{x}-m l \ddot{\theta}=u$
$\left(I+m l^{2}\right) \ddot{\theta}-m g l \theta=m l \ddot{x}$

\section{Diseño de los Controladores para el Sistema Péndulo Invertido}

El controlador difuso para el sistema péndulo invertido se diseña para que opere tal y como lo haría un experto humano quien decide intuitivamente que información usar como entradas para tomar decisiones en el proceso. 
Se usa el error (e) y la derivada del error $(d e / d t)$ como variables de entrada y la fuerza $u$ como variable de salida como se observa en el diagrama de bloques de la Figura 4. La referencia del sistema de control es la posición vertical. Las variables lingüísticas error, derror y FUERZA representadas por $e$, de/dt y $u$ respectivamente, describen los valores lingüísticos que las variables toman y que cambian dinámicamente con el tiempo. Para el caso del péndulo invertido se etiqueta que el error, derror y fuerza toman los valores na (valor negativo alto), $\boldsymbol{n b}$ (valor negativo bajo), $\boldsymbol{c}$ (valor cero), $\boldsymbol{p b}$ (valor positivo bajo) y pa (valor positivo alto).

Para el diseño del controlador se usan funciones de membresía triangulares y trapezoidales, por lo tanto el mecanismo de inferencia implementa el conjunto de reglas IFTHEN del error, de la derivada del error y de la FUERZA, están divididos en cinco conjuntos difusos, la Figura 5 muestra las tres funciones de membresía del sistema del péndulo invertido. Para la posición angular, que es el objetivo de control, las funciones de membresía están definidas como: error $[-0.4,0.4]$, derror $[-20,20]$ y FUERZA $[-300,300]$.

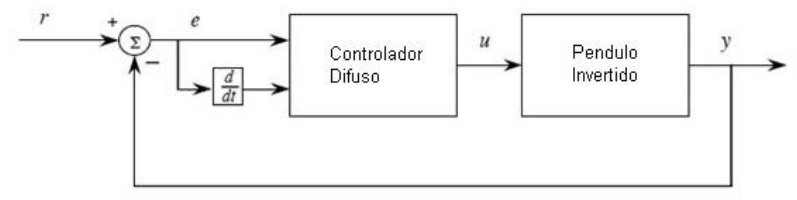

Figura 4 Diagrama de bloques del sistema péndulo invertido con control difuso

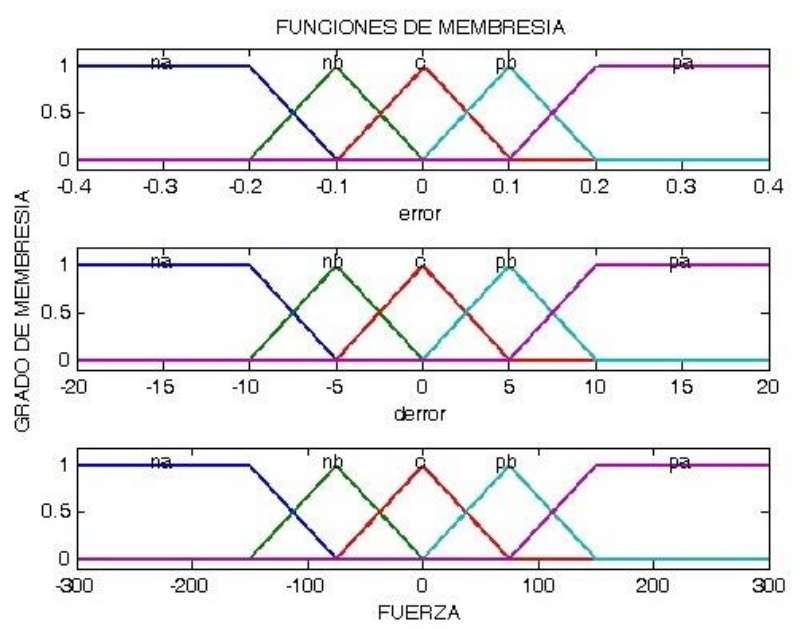

Figura 5 Funciones de membresía del error, derror y fuerza
Dados los valores lingüísticos para el error, derivada del error y Fuerza, se presenta en forma matricial al conjunto de las reglas que definen el funcionamiento del controlador, tal y como lo muestra la Tabla 1, en ella se resumen todas las posibles combinaciones de valores linguísticos en las reglas. Con base a la matriz de reglas y usando (1) se estructuran los enunciados de la base de reglas para el mecanismo de inferencia, obteniendo:

\section{R1: SI error es na Y derror es na ENTONCES fuerza es na}

R2: SI error es $\boldsymbol{n} \boldsymbol{a}$ Y derror es $\boldsymbol{n} \boldsymbol{b}$ ENTONCES fuerza es na

\section{R13: SI error es $\boldsymbol{c}$ Y derror es $\boldsymbol{c}$ ENTONCES fuerza es $c$}

R24: SI error es $\boldsymbol{p a}$ Y derror es $\boldsymbol{p} \boldsymbol{b}$ ENTONCES fuerza es pa

R25: SI error es pa Y derror es pa ENTONCES fuerza es pa

\begin{tabular}{|c|c|c|c|c|c|c|}
\hline \multirow{2}{*}{\multicolumn{2}{|c|}{ Fuerza }} & \multicolumn{5}{|c|}{ Derror } \\
\hline & & $\mathbf{N A}$ & NB & $\mathrm{C}$ & PB & $\mathbf{P A}$ \\
\hline \multirow{5}{*}{ Error } & NA & $\begin{array}{l}\text { NA } \\
\text { R1 }\end{array}$ & $\begin{array}{l}\text { NA } \\
\text { R2 }\end{array}$ & $\begin{array}{l}\text { NA } \\
\text { R3 }\end{array}$ & $\begin{array}{l}\text { NB } \\
\text { R4 }\end{array}$ & $\begin{array}{l}\mathrm{C} \\
\mathrm{R} 5\end{array}$ \\
\hline & NB & $\begin{array}{l}\text { NA } \\
\text { R6 }\end{array}$ & $\begin{array}{l}\text { NA } \\
\text { R7 }\end{array}$ & $\begin{array}{l}\text { NB } \\
\text { R8 }\end{array}$ & $\begin{array}{l}\mathrm{C} \\
\text { R9 }\end{array}$ & $\begin{array}{l}\text { PB } \\
\text { R10 }\end{array}$ \\
\hline & C & $\begin{array}{l}\text { NA } \\
\text { R11 }\end{array}$ & $\begin{array}{l}\text { NB } \\
\text { R12 }\end{array}$ & $\begin{array}{l}\mathrm{C} \\
\mathrm{R} 13\end{array}$ & $\begin{array}{l}\text { PB } \\
\text { R14 }\end{array}$ & $\begin{array}{l}\text { PA } \\
\text { R15 }\end{array}$ \\
\hline & PB & $\begin{array}{l}\text { NB } \\
\text { R16 }\end{array}$ & $\begin{array}{l}\mathrm{C} \\
\text { R17 }\end{array}$ & $\begin{array}{l}\text { PB } \\
\text { R18 }\end{array}$ & $\begin{array}{l}\text { PA } \\
\text { R19 }\end{array}$ & $\begin{array}{l}\text { PA } \\
\text { R20 }\end{array}$ \\
\hline & PA & $\begin{array}{l}\mathrm{C} \\
\mathrm{R} 21\end{array}$ & $\begin{array}{l}\text { PB } \\
\text { R22 }\end{array}$ & $\begin{array}{l}\text { PA } \\
\text { R23 }\end{array}$ & $\begin{array}{l}\text { PA } \\
\text { R24 }\end{array}$ & $\begin{array}{l}\text { PA } \\
\text { R25 }\end{array}$ \\
\hline
\end{tabular}

Tabla 1 Matriz De Reglas Para Péndulo Invertido

Por otra parte, el control PID es de naturaleza lineal, lo que implica que solo puede ser aplicado a sistemas lineales, en el caso del péndulo invertido se aplica al modelo linealizado, por lo que para sintonizar este controlador se requiere obtener la función de transferencia, como se muestra en (11).

$$
\frac{\theta(s)}{U(s)}=\frac{\frac{m l}{q} s}{s^{3}+\frac{b\left(I+m l^{2}\right)}{q_{1}} s^{2}-\frac{(M+m) m g l}{q_{1}} s-\frac{b m g l}{q_{1}}}
$$

Donde:

$$
q_{1}=\left[(M+m)\left(I+m l^{2}\right)-(m l)^{2}\right]
$$

En (11) se muestra la relación que existe entre la posición angular y la fuerza del sistema linealizado, como entrada. Para este trabajo se consideran los siguientes datos del sistema [12]: 


$\begin{array}{lll}\text { M } & \text { Masa del carro } & 0.5 \mathrm{Kg} \\ \mathrm{m} & \text { Masa del péndulo } & 0.2 \mathrm{Kg} \\ \mathrm{b} \quad \text { Fricción del carro } & 0.1 \mathrm{~N} / \mathrm{m} / \mathrm{seg} \\ \mathrm{g} \quad \text { Gravedad } & 9.8 \mathrm{~m} / \mathrm{s}^{\wedge} 2 \\ \mathrm{I} \quad \text { Inercia del péndulo } & 0.006 \mathrm{Kg}^{*} \mathrm{~m}^{\wedge} 2 \\ 1 \quad \text { Longitud al centro de } & 0.3 \mathrm{~m} \\ \text { gravedad del péndulo } & \end{array}$

La función de transferencia en lazo abierto con los datos antes mencionados es la siguiente:

$$
\frac{\theta(s)}{U(s)}=\frac{4.545 s}{s^{3}+0.1818 s^{2}-26.64 s-4.455}
$$

Para el cálculo de las ganancias del controlador se utiliza el método de ZieglerNichols, basado en la ganancia crítica y el periodo crítico [13]. Como se conoce el modelo matemático, se utiliza el lugar de las raíces para la obtención de la ganancia crítica y el periodo crítico. Los valores de las ganancias del controlador PID son:

$$
\begin{array}{ll}
\text { Ganancia proporcional } K_{p}: & 431.3853 \\
\text { Ganancia integral } K_{i}: & 4119.4 \\
\text { Ganancia derivativa } K_{d}: & 9.0349
\end{array}
$$

\section{Resultados. (Análisis de estabilidad)}

La estabilidad es una característica importante que se debe considerar en el desarrollo y análisis de un controlador, cuando el sistema es lineal se dispone de algunos criterios, como el criterio de estabilidad de Routh o el de Nyquist, desafortunadamente cuando el sistema es no lineal estos criterios no son aplicables [14].

Los controladores difusos pertenecen a la familia de los sistemas no lineales, ya que posee una característica de transferencia no lineal, consecuencia de la forma de cómo son disparadas, interpretadas y combinadas las reglas de control [15]. Asimismo, al tratarse de sistemas no lineales, el comportamiento dinámico de los sistemas difusos será más detallado y complejo que el de los sistemas lineales. En esta sección se reporta el análisis de estabilidad realizado por dos métodos, uno conocido como trayectoria lingüística [16], en donde el sistema será estable si la trayectoria lingüística converge al punto de equilibrio [17],

\begin{tabular}{|c|c|c|c|c|c|c|}
\hline \multirow{2}{*}{\multicolumn{2}{|c|}{ FUERZA }} & \multicolumn{5}{|c|}{ DERROR } \\
\hline & & NA & NB & c & PB & PA \\
\hline \multirow{9}{*}{ ERROR } & \multirow[t]{2}{*}{ NA } & NA & NA & NA & NB & C \\
\hline & & $\frac{\mathrm{R} 1}{\mathrm{NA}}$ & R2 & $\frac{\mathrm{R} 3}{\mathrm{~N}}$ & $\mathrm{R} 4$ & $\frac{R 5}{P B}$ \\
\hline & NB & $\mathrm{R} 6$ & 7 & R8 & $\mathrm{Rg}$ & R10 \\
\hline & \multirow{2}{*}{ c } & NA & B & \multicolumn{2}{|c|}{$\mathrm{PB}$} & PA \\
\hline & & R11 & R12 & $\mathrm{R} 13$ & R14 & R15 \\
\hline & \multirow{2}{*}{ PB } & NB & c & PB & PA & PA \\
\hline & & R16 & R17 & R18 & R19. & $\mathrm{R} 20$ \\
\hline & \multirow{2}{*}{ PA } & $\mathrm{c}$ & PB & PA & PA & PA \\
\hline & & R21 & R22 & R23 & R24 & R25 \\
\hline
\end{tabular}
y el otro por medio del análisis de la trayectoria sobre los planos de fase, en donde se grafica el desplazamiento angular contra su velocidad.
Figura 6 Trayectoria lingüística del controlador difuso para el péndulo invertido con perturbación de función impulso.

Para el análisis de estabilidad del controlador difuso, sobre el modelo no lineal, por medio de la trayectoria lingüística se emplea el espacio de estados y se observan las reglas que se activan durante la simulación para crear la trayectoria como lo muestra la Figura 6, dónde se observa que la activación inicia en la regla 13 justo antes de la perturbación, cuando se tiene la perturbación la primera regla que se activa es la 12 , posteriormente se activa la regla 7 , la 8,9 , $14,13,14$ para converger en la regla 13 donde el error y la derivada del error es cero y por supuesto la salida es cero.

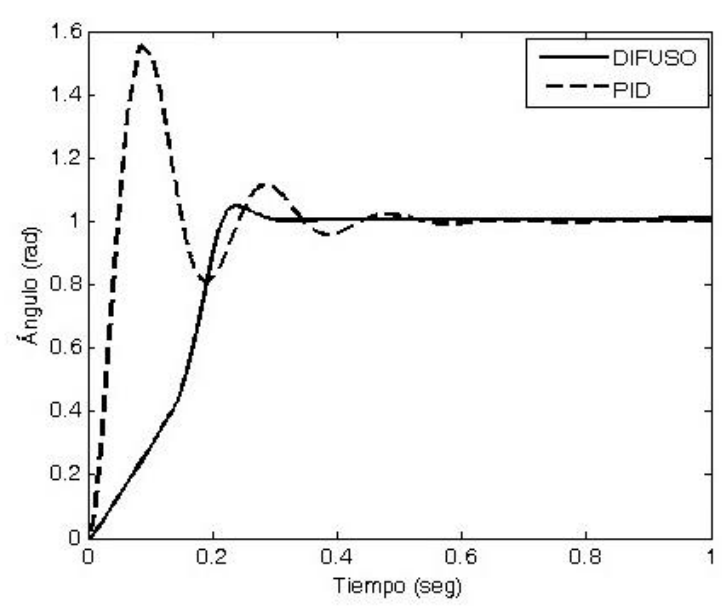

Figura 7 Respuesta transitoria del para una entrada escalón unitario del sistema lineal con el controlador difuso y PID

Para observar las características de desempeño de los sistemas de control sobre el modelo lineal, se obtiene la respuesta transitoria para la entrada escalón unitario. En la Figura 7 se muestran las respuestas transitorias con el controlador difuso y con el PID, aquí se observa que la respuesta con el controlador difuso presenta un tiempo se asentamiento y sobrepaso considerablemente menor que la del controlador PID, el cual llega a alcanzar un sobrepaso cercano al $60 \%$. 
En la otra alternativa para verificar la estabilidad del sistema, por medio del análisis gráfico con el plano de fase, donde se grafica la posición angular contra su velocidad, para la respuesta del sistema con entrada escalón unitario. En la Figura 8, se muestran los planos de fase del sistema lineal y el no lineal, ambos con el mismo controlador difuso, en ella se observa que la trayectoria parte desde un punto establecido por las condiciones iniciales, para este caso son cero, posteriormente las dos respuestas siguen aproximadamente la misma trayectoria que converge a uno, ya que se ha perturbado con un escalón unitario. Por lo que es fácil apreciar que el controlador difuso tiene aproximadamente el mismo efecto de convergencia al punto de estado estable y rapidez de respuesta, tanto en el sistema lineal como en el no lineal.

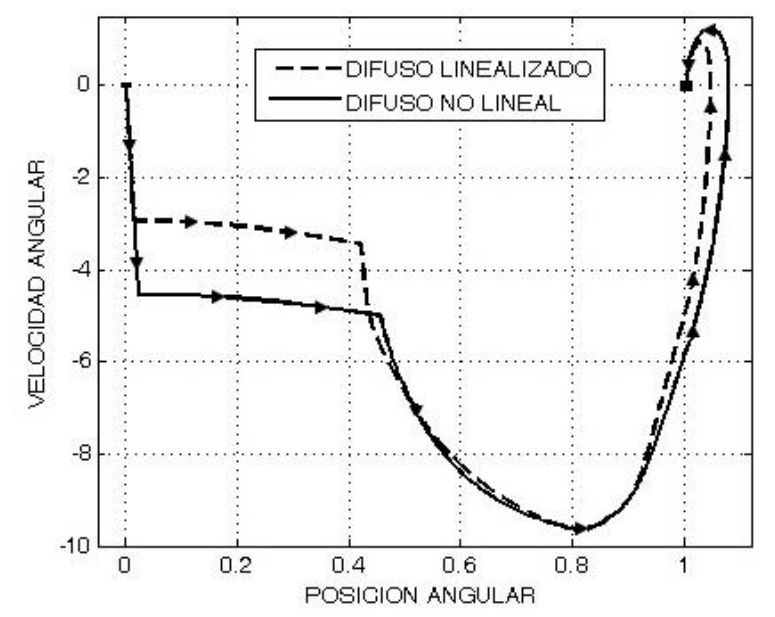

Figura 8 Plano de fase del sistema con control difuso y una entrada escalón

En el mismo sentido, en la Figura 9 se hace la comparación en el plano de fase de la trayectoria de las respuestas del sistema lineal con controlador difuso y con PID como referencia para una entrada escalón unitario. Se observa que la trayectoria descrita por la respuesta del sistema con controlador difuso presenta una convergencia inmediata al valor esperado, mientras que la del PID alcanza valores altos en su recorrido hacia el valor de convergencia, lo que es indicativo del alto valor del sobrepaso y la relativamente extensa duración del transitorio. Sin embargo, es evidente que ambas trayectorias convergen al valor esperado.

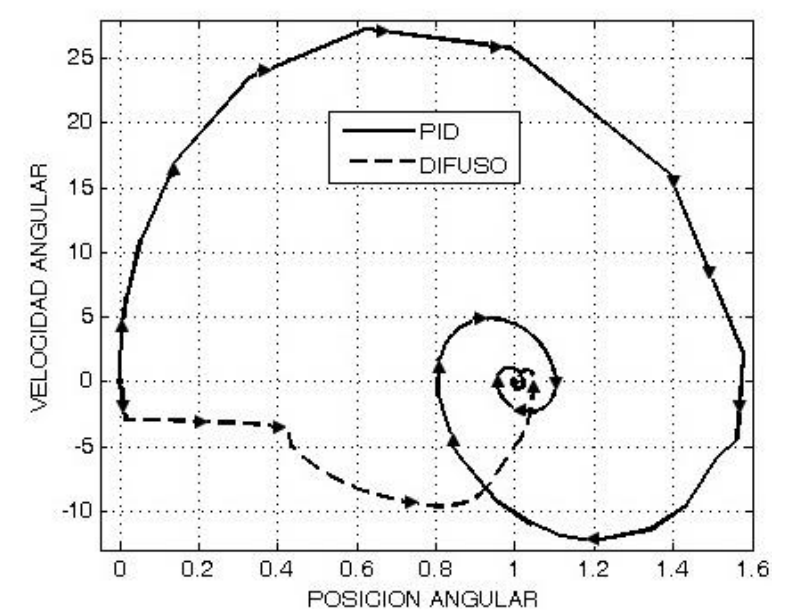

Figura 9 Plano de fase del sistema con control Difuso y control PID aplicado al modelo lineal

\section{Agradecimiento}

Los autores desean expresar su agradecimiento a la carrera de Ingeniería Eléctrica del Instituto Tecnológico Superior de Huauchinango por el apoyo y las facilidades para el desarrollo de este trabajo.

\section{Conclusiones}

En este presente trabajo se desarrolla un controlador difuso para un sistema carropéndulo invertido, el cual es representado por un modelo no lineal, del que se obtiene un modelo equivalente linealizado bajo la consideración de pequeñas oscilaciones. Se obtiene el comportamiento dinámico del sistema con una entrada impulso unitario, para dos casos: en el primero, los dos modelos con controlador difuso, las respuestas obtenidas muestran que el sistema no lineal es ligeramente más rápido pero con un sobreaso superior. En el segundo caso se comparan las respuestas del controlador difuso contra la del PID, sobre el modelo lineal; de donde la respuesta del controlador difuso presenta un menor sobrepaso, mayor rapidez y menor tiempo de asentamiento.

En la comparación de las características de desempeño de los controladores difuso y PID, sobre el modelo lineal con entrada escalón unitario. El controlador difuso presenta mejores características de desempeño, ya que en las respuestas obtenidas, se observa un tiempo de asentamiento y sobrepaso considerablemente menor que el controlador PID. La principal aportación de la investigación es el análisis de estabilidad, con trayectorias linguísticas, del sistema no lineal con controlador difuso. 
Con el cual se obtiene, que trayectoria siempre se mantiene cerca del valor esperado activando únicamente las reglas que representan valores cercanos a cero, tanto para el error como para su derivada, observándose que finalmente dicha trayectoria converge rápidamente a cero en las dos variables, lo que indicando que se ha alcanzado el valor de referencia establecido.

Para el método de estabilidad por medio de plano de fase, donde se hace la comparación entre las trayectorias de la respuesta del sistema lineal con controlador difuso y con el controlador PID, es claro que la respuesta del difuso presenta una trayectoria más directa en comparación con la del PID, lo que indica el bajo tiempo de asentamiento que presenta el este controlador. Aun así ambas respuestas convergen al mismo valor de referencia. La investigacion aporta de manera contudente la robustez de un control difuso para sistemas no lineales comparado con tecnicas tradicionales, ademas de una metodologia altenativa para el analisis de la estabilidad del mismo.

\section{Anexo}

\section{Simulación del sistema péndulo invertido}

La simulación del sistema péndulo invertido con controlador se desarrolla sobre el software MATLAB/SIMULINK empleando la herramienta FUZZY LOGIC TOOLBOX, la cual permite realizar pruebas y simulaciones, además de ver resultados de forma gráfica. Para esta prueba se utiliza una señal impulso simulando una perturbación de 1 N. En la Figura 10 se muestra el sistema en SIMULINK con el controlador difuso y con el PID.

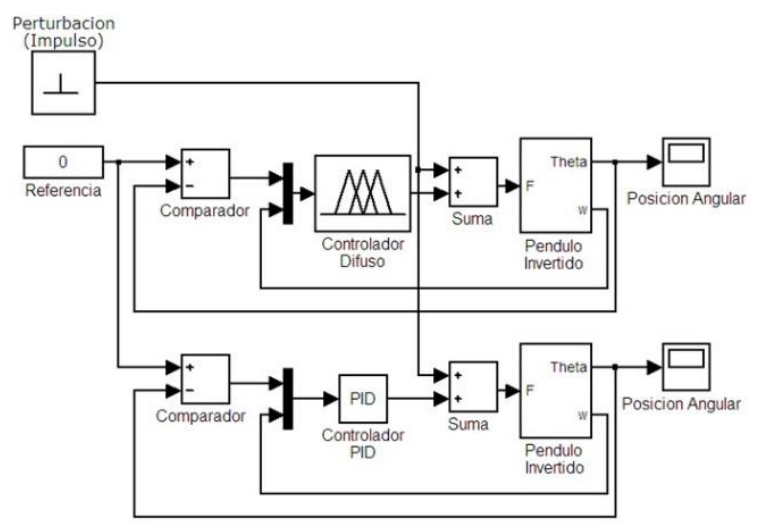

Figura 10 Sistema de control realimentado control difuso y PID en SIMULINK
La Figura 11, muestra el comportamiento de la respuesta del péndulo invertido ante una perturbación impulso de $1 \mathrm{~N}$ y una señal de referencia de cero con respecto al eje vertical, cuando es controlado por el Controlador Difuso y el Controlador PID, tanto en el modelo no lineal, como en el linealizado. La respuesta que se está graficando corresponde a la posición angular $\theta$ con respecto a la vertical y la posición del carro se dejó evolucionar libremente.

En la Figura 11 A) se comparan las respuestas del sistema linealizado con controlador difuso y del control PID. Para este caso al aplicar el control difuso se obtiene un sobrepaso de $2.22 \%$ y un tiempo se asentamiento $0.3520 \mathrm{seg}$, teniendo una respuesta rápida con respecto al PID, que tiene un sobrepaso de $4.94 \%$ que es un valor alto comparado con el difuso y un tiempo de asentamiento de 0.6775 seg, en la Figura 11 B) se comparan las respuestas del sistema no lineal y el linealizado, ambos con el controlador difuso. En el modelo no lineal se tiene un sobrepaso de $2.62 \%$ que es un valor mayor al del modelo linealizado que es de $2.22 \%$, pero su respuesta es lenta, debido a las limitaciones que tiene al linealizarse.
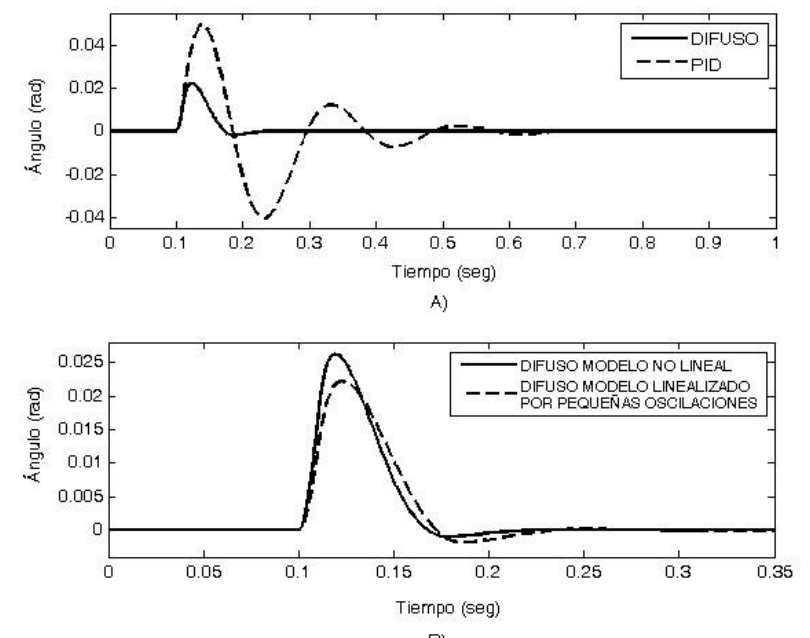

Figura 11 Respuesta del sistema con una perturbación de $1 \mathrm{~N}$

\section{Referencias}

[1] Dorf, Richard C.y Bishop Robert H., Sistemas de Control Moderno, 10. Ed., PrenticeHall, 2007.

[2] H. J. Marquez. Nonlinear control systems. Analysis and design. John Wiley \& Sons,Inc., 2003.

[3] S. Sastry. Nonlinear system: analysis, stability, and control. Springer, New York, 1999. 
[4] Kılıç, E., Özçalık, H.R., Yılmaz, Ş, Şit, S,. “A Comparative Analysis of FLC and ANFIS Controller for Vector Controlled Induction Motor Drive." ACEMP - OPTIM ELECTROMOTION JOINT CONFERENCE. 2015.

[5] Kılıç, E., Özcalık, H.R., Yılmaz, Ş, Gani A,. "Isıl İşlem Uygulamalarında Kullanılan Bir Karışım Tankının Bulanık Mantık Esaslı Denetimi." Kahramanmaras Sutcu Imam University Journal of Engineering Sciences (KSU-JES), Cilt: 17 Sayı:1, 16-21. 2014

[6] Özçalık, H.R., Kılıç, E., Yıılmaz, Ş., Gani, A. "Bulanık Mantık Esaslı Sivi Seviye Denetiminde Farklı Üyelik Fonksiyonlarının Denetim Performansına Etkisinin İncelenmesi." Otomatik Kontrol Ulusal Toplantıs1, pp.243247. 2013

[7] Özçalık, H. R., Yıldız, C., Danaci, M., Koca, Z. "RBF Based Induction Motor Control with a Good Nonlinearity Compensation." Computational and Ambient Intelligence Volume 4507 of the series Lecture Notes in Computer Science pp 878-886. 2007.

[8] R. Brasilia, V.Sankaranarayanan, Nonlinear control of mobile inverted pendulum, Robotics and Autonomous Systems, 70 (2015), 145-155. https://doi.org/10.1016/j.robot.2015.02.012

[9] M. M. Almeida, G. V. Raffo, Nonlinear Balance Control of an Inverted Pendulum on a Tilt-rotor UAV, IFAC-PapersOnLine, 48 (2015), no. 19, 168-173. https://doi.org/10.1016/j.ifacol.2015.08.078

[10] K. M. Passino and S. Yurkovich. Fuzzy Control. AddisonWesley Longman, Inc., Menlo Park, California, USA, 1998.

[11] L. X. Wang, Adaptive Fuzzy Systems and Control, Englewood Cliffs NJ: Prentice-Hall, 1994.

[12] Mohan Akole, Barjeev Tyagi (2008) "Design of fuzzy logic controller for nonlinear model of inverted pendulum-cart system", XXXII National Systems Conference, Nsc, pp 750-755.

[13] K.Ogata, Ingeniería de Control Moderno, 3a.Ed. Prentice-Hall, 1998.
[14] Leonid Reznik, "Fuzzy Controllers", Newnes, Butterworth-Heinemann Linacre House, Jordan Hill, Oxford OX2 8DP, 1er Ed,, ISBN 075063429 4., 1997.

[15] Lee C. C. (1990), "Fuzzy Logic in Control Systems: Fuzzy Logic Controller (Part I and II)". IEEE Trans. On Systems, Man, and Cybernetics, vol. 20, no. 2, pp. 404-435.

[16] Braae, M. and D. A. Rutherford (1979), "Theoretical and linguistics aspects of the fuzzy logic controller", Automatica, vol. 15, pp. 553577.

[17] Basil M. Al-Hadithi, Fernando Matía y Agustín Jiménez.(2007). "Análisis de estabilidad de sistemas borrosos". Revista Iberoamericana de automática e Informática Industrial. ISSN: 1697-7912, vol 4, no. 2, pp. 725 . 